\title{
MHD Mixed Convection Flow of Viscoelastic Fluid Embedded in Porous Medium
}

\author{
A.R.M.Kasim ${ }^{1}$, N.F.Mohammad ${ }^{1,2}$, Aurangzaib ${ }^{1}$, S. Shafie ${ }^{1}$ \\ ${ }^{1}$ Department of Mathematical Sciences, Faculty of Science, Universiti Teknologi Malaysia. \\ ${ }^{2}$ Department of Computational and Theoretical Sciences, International Islamic University Malaysia, 25200 Kuantan, Pahang, Malaysia. \\ Received 11 December 2012, Revised 11 February 2013, Accepted 19 February 2013, Available online 23 February 2013
}

\begin{abstract}
An analysis has been carried out to investigate the effect of magnetic field presence on the mixed convection boundary layer flow of viscoelastic fluid over a horizontal circular cylinder in a porous medium. The governing non-similar partial differential equations are transformed into dimensionless forms and then solved numerically using the Keller-box method. Some important parameters have been discussed in this study which include the Prandtl number (Pr), magnetic parameter (M), viscoelastic parameter (K), porosity parameter $(\gamma)$ and the mixed convection parameters ( $\lambda$ ). The results show the values of the velocity decrease when the value of viscoelastic parameter increase and the reverse trend were observe for temperature profile. Numerical results of local skin friction as well as local Nusselt number are also presented in tabular form.
\end{abstract}

| MHD | Mixed convection | Porous medium | Viscoelastic | Keller box |

® 2013IbnuSina Institute. All rights reserved. http://dx.doi.org/10.11113/mjfas.v9n1.77

\section{INTRODUCTION}

The interest of flows in viscoelastic fluids has grown considerably because of their applications in engineering and several industrial-manufacturing processes involving petroleum drilling, manufacturing of foods and papers. On the other hand, this type of fluidsalso have applications involving atomization such as paints, coating, inks, jet fuels and possible to reduce frictional drag on the hulls of ships and submarines. The boundary layer theory for viscoelastic fluids, which is similar to second-grade fluids, was developed by a few researchers since the last few decades [1-3]. The differential governing equations of the viscoelastic fluid problems are an order higher than those for the Newtonian (viscous) fluid and the adherence boundary conditions are insufficient to determine the numerical solution completely. Therefore, a boundary condition is needed in addition to the usual adherence boundary conditions [4-7]. Recently, the research on magnetohydrodynamic(MHD) in fluid flow have attracted many researchers due to its potential applications in engineering and industrial fields. Magnetohydrodynamic power generators and accelerators, cooling of nuclear reactors and crystal growth are included in this area. Accordingly, a considerable amount of research has been done on the effects of the electrically conducting fluids such as liquid metals, water mixed with a little acid and others in the presence of transverse magnetic field on the flow and heat transfer characteristics over various geometries. One of MHD research is heat convection in a magnetized electrically conducting layer [8]. The MHD is the science of the motion of electrically conducting fluids under magnetic fields. This situation is essentially one of mutual interaction between the fluid velocity field and the electromagnetic field; the motion affects the magnetic field and the magnetic field affects the motion. Thus, the term MHD attempts to convey this relationship [9]. Some researchers have investigated on MHD flow problems in non-Newtonian fluid focussing on viscoelastic fluid [10-14]. The transport of heat in porous medium is a process involving the application in a broad spectrum of disciplines ranging from chemical engineering to geo-physics. Many metallurgical processes involve the cooling of continuous strips or filaments by drawing them through a quiescent fluid. One of the group researchers have studied heat and mass transfer for Soret and Dufour's effect on mixed convection boundary layer flow over a stretching vertical surface in a porous medium filled with a viscoelastic fluid[15]. In the same year, another group of reseachers carried out the investigation on flow and heat transfer characterictics of viscoelastic fluid in a porous medium over an impermeable stretching sheet with viscous dissipation using the power series method (using Kummer's function) [16]. Very recently, an investigation on analytic solution of homotopy analysis method for MHD viscoelastic fluid flow and heat transfer in a channel with a stretching wall has been done by Raftari and Vajravelu [17]. They obtained the analytical solutions in the form of infinite series and the convergence of the series solution is discussed explicitly. Based to the above review, this present paper aims to study the effects of magnetic field presence on mixed convection boundary layer flow in viscoelastic fluid past a circular cylinder with 
constant temperature in porous medium. The governing equations are solved numerically by using the Keller box method.

\subsection{MATHEMATICAL FORMULATION}

The problem of MHD mixed convection boundary layer flow for an isothermal horizontal circular cylinder placed in a viscoelastic fluid is studied. Figure 1 illustrates the geometry of the problem and the corresponding coordinate system.It is assumed that the constant temperature of the surface of the cylinder is $T_{w}$ and the ambient fluid temperature is $T_{\infty}$, where $T_{w}>T_{\infty}$ corresponds to a heated cylinder (assisting flow) and $T_{w}<T_{\infty}$ corresponds to a cooled cylinder (opposing flow), respectively.

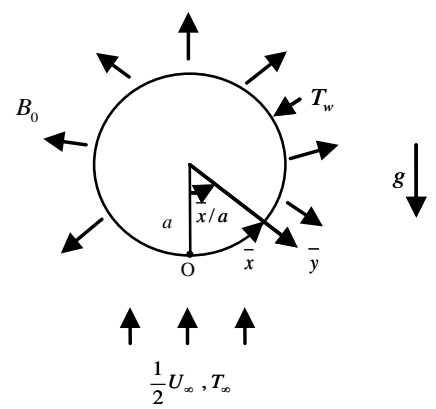

Fig.1.Physical model and coordinate system.

It is assumed that the Boussinesq and boundary layer approximations are valid. Under these assumptions, the equations governing the steady mixed convection boundarylayer flow are;

Continuity equation:

$\frac{\partial \bar{u}}{\partial \bar{x}}+\frac{\partial \bar{v}}{\partial \bar{y}}=0$

Momentum equation:

$\bar{u} \frac{\partial \bar{u}}{\partial \bar{x}}+\bar{v} \frac{\partial \bar{u}}{\partial \bar{x}}=\bar{u}_{e} \frac{d \bar{u}_{e}}{d \bar{x}}+v \frac{\partial^{2} \bar{u}}{\partial \bar{y}^{2}}$

$+\frac{\mathrm{k}_{0}}{\rho}\left[\frac{\partial}{\partial \bar{x}}\left(\bar{u} \frac{\partial^{2} \bar{u}}{\partial \bar{y}^{2}}\right)+\bar{v} \frac{\partial^{3} \bar{u}}{\partial \bar{y}^{3}}+\frac{\partial \bar{u}}{\partial \bar{y}} \frac{\partial^{2} \bar{v}}{\partial \bar{y}^{2}}\right]$

$-\frac{\sigma B_{0}}{\rho}\left(\bar{u}-\bar{u}_{e}\right)-\frac{v}{\mathrm{~K}^{*}}\left(\bar{u}-\bar{u}_{e}\right)+g \beta\left(T-T_{\infty}\right) \sin (\bar{x} / a)$,

Energy equation: $\bar{u} \frac{\partial T}{\partial \bar{x}}+\bar{v} \frac{\partial T}{\partial \bar{y}}=\alpha \frac{\partial^{2} T}{\partial \bar{y}^{2}}$,

subject to the boundary conditions:

$$
\begin{aligned}
& \bar{u}=\bar{v}=0, \quad T=T_{w}, \quad \text { on } \quad \bar{y}=0, \\
& \bar{u}=\bar{u}_{e}(x), \quad \partial \bar{u} / \partial \bar{y}=0, \quad T=T_{\infty}, \quad \text { as } \quad y \rightarrow \infty .
\end{aligned}
$$

The Cartesian coordinates $\bar{x}$ is measured along the surface of the cylinder starting from the lower stagnation point of the cylinder and $\bar{y}$ is the coordinate measured normal to the surface of the cylinder. $\bar{u}$ and $\bar{v}$ are the velocity components along the $\bar{x}$ - and $\bar{y}$-axes and $\bar{u}_{e}(\bar{x})$ is the velocity outside the boundary layer.

The non-dimensional variables are introduced as follows:

$$
\begin{gathered}
x=\bar{x} / a, \quad y=\operatorname{Re}^{1 / 2}(\bar{y} / a), \quad u=\bar{u} / U_{\infty}, \quad v=\operatorname{Re}^{1 / 2}\left(\bar{v} / U_{\infty}\right) \\
u_{e}=\overline{u_{e}}(\bar{x}) / U_{\infty}, \quad \theta=\left(T-T_{\infty}\right) /\left(T_{W}-T_{\infty}\right),
\end{gathered}
$$

where $\operatorname{Re}=U_{\infty} a / v$ is the Reynolds number.

Substituting Equation (5) into Equations (1-4), the following non-dimensional equations are obtained;

Continuity equation:

$\frac{\partial u}{\partial x}+\frac{\partial v}{\partial y}=0$

Momentum equation:

$$
\begin{aligned}
u \frac{\partial u}{\partial x}+ & v \frac{\partial u}{\partial y}=u_{e} \frac{d u_{e}}{d x}+\frac{\partial^{2} u}{\partial y^{2}} \\
+ & K\left[\frac{\partial}{\partial x}\left(u \frac{\partial^{2} u}{\partial y^{2}}\right)+v \frac{\partial^{3} u}{\partial y^{3}}+\frac{\partial u}{\partial y} \frac{\partial^{2} v}{\partial y^{2}}\right] \text { (7) } \\
& -(\mathrm{M}+\gamma)\left(u-u_{e}\right)+\lambda \theta \sin x
\end{aligned}
$$

Energy equation:

$u \frac{\partial \theta}{\partial x}+v \frac{\partial \theta}{\partial y}=\frac{1}{\operatorname{Pr}} \frac{\partial^{2} \theta}{\partial y^{2}}$

subject to the boundary conditions:

$u=v=0, \quad \theta=1, \quad$ at $\quad y=0$,
$u=u_{e}(x), \quad \frac{\partial u}{\partial y}=0, \quad \theta=0, \quad$ as $\quad y \rightarrow \infty$, 
where Pr is the Prandtl number, Gr is the Grashof number, $\mathrm{K}$ is the viscoelastic parameter, $\lambda$ is the mixed convection parameter, $\gamma$ is porosity parameter and Mis the magnetic parameter which are defined as:

$$
\begin{aligned}
& \mathrm{Gr}=\frac{g \not h\left(T_{w}-T_{\infty}\right) a^{3}}{v^{2}}, \quad \mathrm{~K}=\frac{k_{0} U_{\infty}}{a \rho v}, \quad=\frac{\mathrm{Gr}}{\mathrm{Re}^{2}} \\
& \mathrm{M}=\frac{\sigma B_{0} a}{\rho U_{\infty}}, \quad \gamma=\frac{v}{K^{*}} \frac{a}{U_{\infty}}
\end{aligned}
$$

It is noticed that when $\lambda>0$, the problem involves assisting flow $\left(T_{w}>T_{\infty}\right)$ and when $\lambda<0$, the problem involves the opposing flow $\left(T_{w}<T_{\infty}\right)$.

\subsection{SOLUTION PROCEDURE}

Following Merkin [19], it is assumed that $u_{e}(x)=$ $\sin \mathrm{x}$. In order to solve Equations (6-8), the following similarity variables are introduced:

$\psi=x f(x, y), \quad \theta=\theta(x, y)$,

where $\psi$ is the stream function defined in the usual way as:

$u=\frac{\partial \psi}{\partial y}, v=-\frac{\partial \psi}{\partial x}$.

Substituting Equations (11) and (12) into Equations (6-8), the resulting equations are obtained:

$$
\begin{aligned}
& \frac{\partial^{3} f}{\partial y^{3}}+f \frac{\partial^{2} f}{\partial y^{2}}-\left(\frac{\partial f}{\partial y}\right)^{2}+\frac{\sin x \cos x}{x} \\
& +\lambda \frac{\sin x}{x} \theta+\mathrm{K}\left\{2 \frac{\partial f}{\partial y} \frac{\partial^{3} f}{\partial y^{3}}-f \frac{\partial^{4} f}{\partial y^{4}}-\left(\frac{\partial^{2} f}{\partial y^{2}}\right)^{2}\right. \\
& \left.+x\left(\frac{\partial^{2} f}{\partial x \partial y} \frac{\partial^{3} f}{\partial y^{3}}-\frac{\partial f}{\partial x} \frac{\partial^{4} f}{\partial y^{4}}+\frac{\partial f}{\partial y} \frac{\partial^{4} f}{\partial x \partial y^{3}}-\frac{\partial^{2} f}{\partial y^{2}} \frac{\partial^{3} f}{\partial x \partial y^{2}}\right)\right\} \\
& -(\mathrm{M}+\gamma)\left(\frac{\partial f}{\partial y}-\frac{\sin x}{x}\right)=x\left(\frac{\partial f}{\partial y} \frac{\partial^{2} f}{\partial x \partial y}-\frac{\partial f}{\partial x} \frac{\partial^{2} f}{\partial y^{2}}\right), \\
& \frac{1}{\operatorname{Pr}} \frac{\partial^{2} \theta}{\partial y^{2}}+f \frac{\partial \theta}{\partial y}=x\left(\frac{\partial f}{\partial y} \frac{\partial \theta}{\partial x}-\frac{\partial f}{\partial x} \frac{\partial \theta}{\partial y}\right),
\end{aligned}
$$

subject to the boundary conditions:

$$
\begin{aligned}
& f=\frac{\partial f}{\partial y}=0, \quad \theta=1, \quad \text { at } \quad y=0, \\
& \frac{\partial f}{\partial y}=\frac{\sin x}{x}, \quad \frac{\partial^{2} f}{\partial y^{2}}=0, \quad \theta=0, \quad \text { as } \quad y \rightarrow \infty .
\end{aligned}
$$

At the lower stagnation point of the cylinder $(x \approx 0)$, Equations (12) and (13) are reduced into the following ordinary differential equations:

$$
\begin{gathered}
f^{\prime \prime}+f f^{\prime \prime}-f^{\prime 2}+1-(\mathrm{M}+\gamma)\left(f^{\prime}-1\right)+\lambda \theta \\
+\mathrm{K}\left(2 f^{\prime} f^{\prime \prime}-f f^{i v}-f^{\prime 2}\right)=0 \\
\frac{1}{\mathrm{Pr}} \theta^{\prime \prime}+f \theta^{\prime}=0, \quad \text { (17) }
\end{gathered}
$$

with the boundary conditions:

$$
\begin{aligned}
& f(0)=f^{\prime}(0)=0, \quad \theta(0)=1, \\
& f^{\prime}(\infty)=1, \quad f^{\prime \prime}(\infty)=0, \quad \theta(\infty)=0 .
\end{aligned}
$$

In practical applications, the physical quantities of principal interest are the local skin friction coefficient and the local Nusselt number, which are defined as

$C_{f x}=x f^{\prime \prime}(0), \quad N u_{x}=-\theta^{\prime}(0)$.

\subsection{RESULTS AND DISCUSSION}

Equations (13) and (14) together with Equations (16) and (17) which were subjected to boundary conditions (15) and (18), respectively have been solved numerically using an efficient implicit finite-difference method known as the Keller-box scheme by Cebeci and Bradshaw (1988). The solution is obtained in the following four steps: discretize the governing equations using finite difference method, linearize the resulting algebraic equations by Newton's method, write them in matrix-vector form and finally, solve the linear system by the block-tridiagonal-elimination technique. The numerical solution is obtained at some $x$ around the cylinder starting from the forward stagnation point $(x \approx 0)$ for some values of mixed convection parameter, $\lambda$, magnetic parameter, $\mathrm{M}$, porosity parameter, $\gamma$ and viscoelastic parameter, K. The step size for $x$ and $\eta$ is 0.01 and 0.025 , respectively. At every point, the iteration process goes on until the convergence criterion for all the variables, $10^{-6}$, is attained.

An extension of previous works has been performed to investigate the heat transfers of a viscoelastic fluid flow over a horizontal circular cylinder which includes the magnetic effect in porous medium. In order to check the validity of the present work, the values of $N u_{x}$ and $C_{f x}$ as given by Equation (19) are compared with those of Anwar et al.[7]in Table 1 for $\mathrm{M}=0, \gamma=0$ at $\operatorname{Pr}=1$ with $\lambda=0$ and 1 and the various values of viscoelastic parameter, K. It is seen that the results are found to be in excellent agreement. Therefore, the validity of the present results is proven to be in parallel with existing work.

Figs. 2 and 3 show the distribution of velocity and temperature profile respectively. It shows that, an increase 
in the porosity parameter, $\gamma$ leads to decrease in the velocity and increase the temperature profile. Further, Figs. 4 and 5 show the distribution of velocity and temperature profile respectively. It can be seen that, the velocity is increased by increasing the value of magnetic field, $M$, while opposite trend is shown for the temperature profile.

Table 1.Comparison of values of $f^{\prime \prime}(0)$ and $-\theta^{\prime}(0)$ for various values of $\mathrm{K}$ when $\lambda=0$ and 1 and for $\operatorname{Pr}=1$ and $M=0$.

\begin{tabular}{|c|c|c|c|c|c|c|c|c|}
\hline \multirow{3}{*}{ K } & \multicolumn{2}{|c|}{ Anwar et al.[7] } & \multicolumn{2}{|c|}{ Present result } & \multicolumn{2}{|c|}{ Anwar et al.[7] } & \multicolumn{2}{|c|}{ Present result } \\
\hline & \multicolumn{4}{|c|}{$\lambda=0$} & \multicolumn{4}{|c|}{$\lambda=1$} \\
\hline & $f^{\prime \prime}(0)$ & $-\theta^{\prime}(0)$ & $f^{\prime \prime}(0)$ & $-\theta^{\prime}(0)$ & $f^{\prime \prime}(0)$ & $-\theta^{\prime}(0)$ & $f^{\prime \prime}(0)$ & $-\theta^{\prime}(0)$ \\
\hline 0 & 1.232632 & 0.570519 & 1.232632 & 0.570519 & 1.736738 & 0.615601 & 1.736738 & 0.615601 \\
\hline 0.01 & 1.221447 & 0.569130 & 1.221447 & 0.569130 & 1.718552 & 0.613861 & 1.718552 & 0.613861 \\
\hline 0.1 & 1.134172 & 0.558175 & 1.134172 & 0.558175 & 1.580229 & 0.600089 & 1.580229 & 0.600089 \\
\hline 0.2 & 1.058180 & 0.548077 & 1.058180 & 0.548077 & 1.464141 & 0.587800 & 1.464141 & 0.587800 \\
\hline 0.4 & 0.945907 & 0.532036 & 0.945907 & 0.532036 & 1.298364 & 0.568851 & 1.298364 & 0.568851 \\
\hline 0.6 & 0.864985 & 0.519487 & 0.864985 & 0.519487 & 1.182212 & 0.554389 & 1.182212 & 0.554389 \\
\hline 0.7 & 0.832019 & 0.514101 & 0.832019 & 0.514101 & 1.135550 & 0.548256 & 1.135550 & 0.548256 \\
\hline 0.9 & 0.776511 & 0.504626 & 0.776511 & 0.504626 & 1.057711 & 0.537559 & 1.057711 & 0.537559 \\
\hline 1 & 0.752803 & 0.500411 & 0.752803 & 0.500411 & 1.024719 & 0.532833 & 1.024719 & 0.532833 \\
\hline 2 & 0.596874 & 0.469671 & 0.596874 & 0.469671 & 0.810695 & 0.498821 & 0.810695 & 0.498821 \\
\hline 3 & 0.510914 & 0.449922 & 0.510914 & 0.449922 & 0.694301 & 0.477261 & 0.694301 & 0.477261 \\
\hline 4 & 0.454295 & 0.435499 & 0.454295 & 0.435499 & 0.617991 & 0.461601 & 0.617991 & 0.461601 \\
\hline 5 & 0.413321 & 0.424228 & 0.413321 & 0.424228 & 0.562865 & 0.449394 & 0.562865 & 0.449394 \\
\hline 10 & 0.303669 & 0.389777 & 0.303669 & 0.389777 & 0.415342 & 0.412127 & 0.415342 & 0.412127 \\
\hline
\end{tabular}

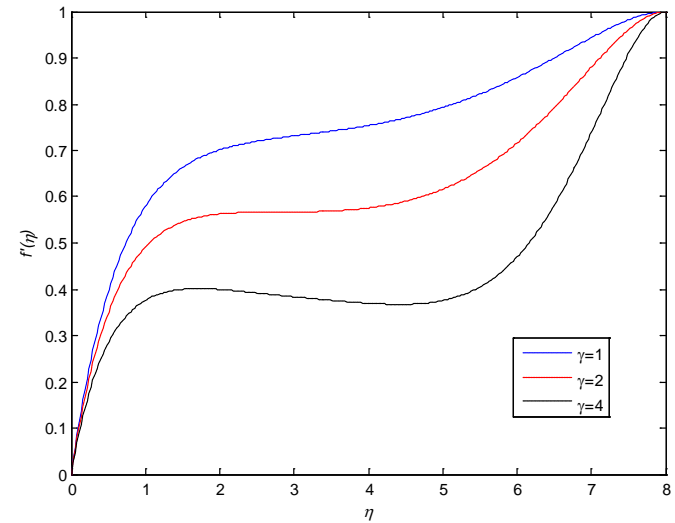

Fig.2. Velocity profile for $\lambda=1, \operatorname{Pr}=1, \mathrm{~K}=1 \mathrm{M}=1$ and various values of $\gamma$ (porosity parameter)

As we can see for the large value of magnetic field, the profile of velocity and temperature did not show any difference. We can say that the magnetic effect is not good enough for a larger magnetic force.As illustrated in

Figs. 6 and 7, as mixed convection parameter increases, we found that the velocity profiles also increase while the temperature profiles decrease. The velocity and temperature profiles for various value of visoelastic parameter, $\mathrm{K}$ are plotted in Figs. 8 and 9 respectively. From these figures it can be seen that, the values of the velocity decrease when the value of viscoelastic parameter increase and the reverse trend were observe for temperature profile. This behaviour is also similar to the problem of stagnation-point flow of a viscoelastic fluid towards a stretching surface investigated by Mahapatra and Gupta [18].

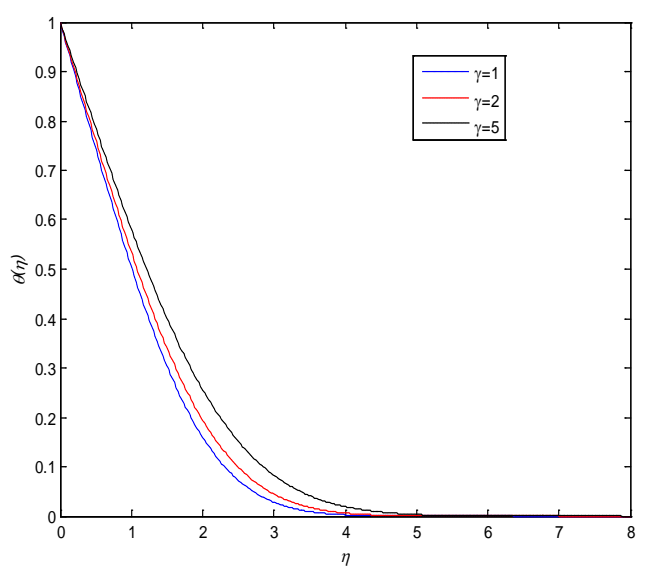

Fig. 3. Temperature profile for $\lambda=1, \operatorname{Pr}=1, \mathrm{~K}=1 \mathrm{M}=1$ and various values of $\gamma$ (porosity parameter)

\section{CONCLUSION}

A steady two-dimensional mixed convection of an incompressible viscoelastic mixed convection boundary layer in porous medium in the presence of magnetic field was studied. The governing boundary layer equations are 
transformed into non-dimensional form and the resulting nonlinear system of partial differential equations was solved numerically using the Keller-box method. The comparison of the numerical results of local skin friction and local Nusselt number shows an excellent agreement with previous publication. Present results show that, as both mixed convection and magnetic parameter increase, the distribution of velocity decreased while the temperature increased. Moreover, the numerical values of local skin friction, local Nusselt number and both distribution of velocity and temperature profile vary with increasing viscoelastic and porosity parameter.

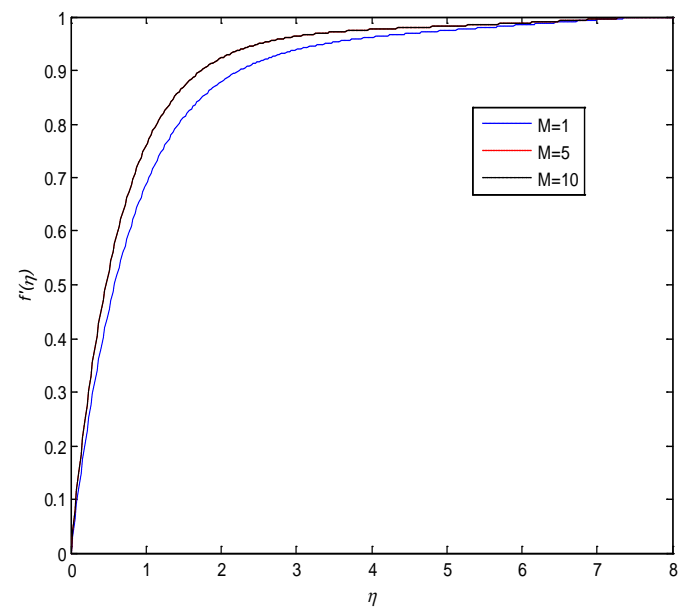

Fig. 4.Velocity profile for $\lambda=1, \operatorname{Pr}=1, \mathrm{~K}=1 \gamma=0.1$ and various values of $\mathrm{M}$ (magnetic parameter)

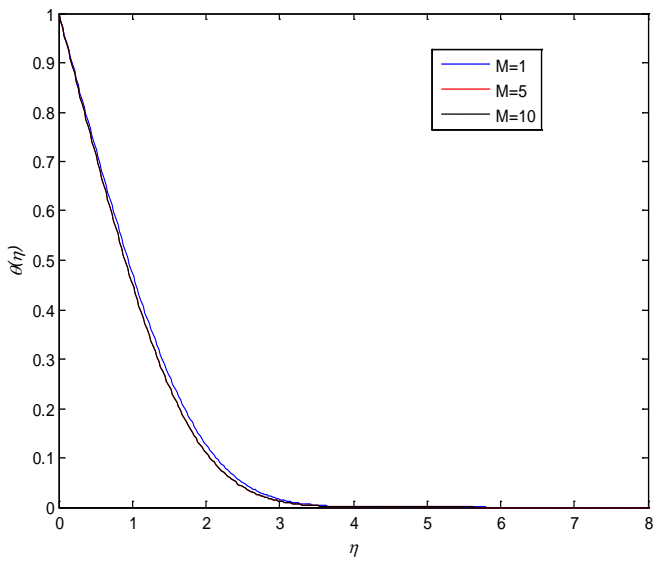

Fig. 5. Temperature profile for $\lambda=1, \operatorname{Pr}=1, K=1$, $\gamma=0.1$ and various values of $\mathrm{M}$ (magnetic parameter)

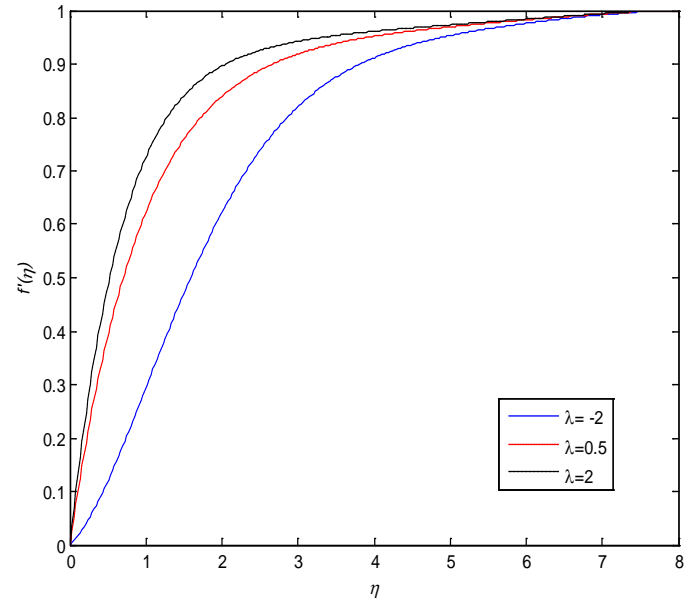

Fig. 6. Velocity profile for $\mathrm{M}=0.5, \operatorname{Pr}=1, \mathrm{~K}=1 \gamma=0.1$ and various values of $\lambda$ (mixed convection parameter)

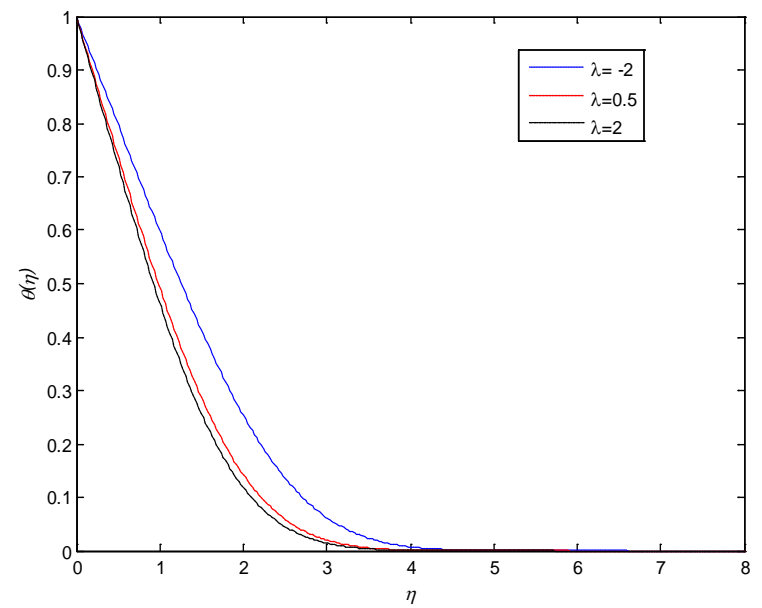

Fig. 7. Temperature profile for $\mathrm{M}=0.5, \operatorname{Pr}=1, \mathrm{~K}=1$, $\gamma=0.1$ and various values of $\lambda$ (mixed convection parameter)

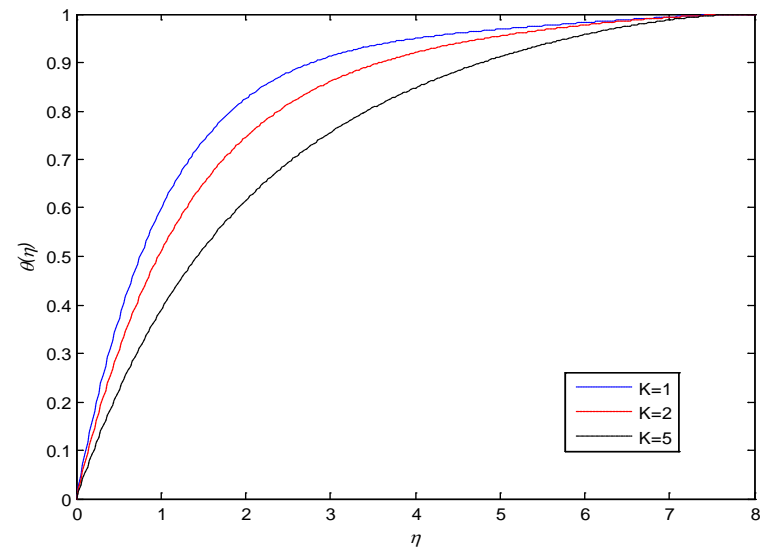

Fig. 8. Velocity profile for $\mathrm{M}=0.5, \operatorname{Pr}=1, \lambda=1 \gamma=0.1$ and various values of $\mathrm{K}$ (viscoelastic parameter) 


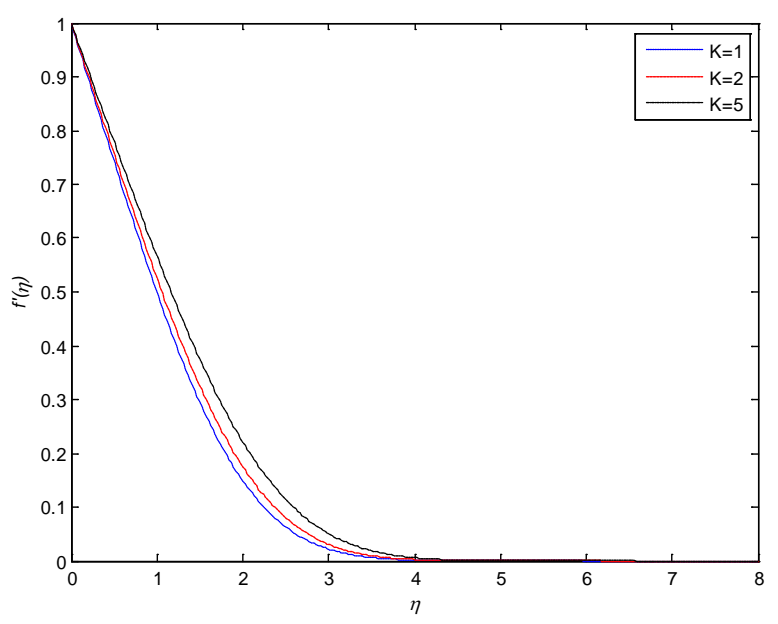

Fig. 9. Temperature profile for $\mathrm{M}=0.5, \operatorname{Pr}=1, \lambda=1 \gamma$ $=0.1$ and various values of $\mathrm{K}$ (viscoelastic parameter)

\section{ACKNOWLEDGEMENT}

This work is supported by a research grant (Vot FRGS No. 4F109, 02H80\& 04H27) from Universiti Teknologi Malaysia, UTM, and MOHE.

\section{REFERENCES}

[1] J.G. Oldroyd, Proceedings of the Royal Society of London. Series A, Mathematical and Physical Sciences 200 (1950) 523-541

[2] D.W. Beard, K. Walters, Proc. Camb. Phil. Soc. , (1964) 667-674

[3] K.R. Rajagopal, J. Non-Newtonian Fluid Mech., ( 1984) 239-246.

[4] A.R.M. Kasim, N.F. Mohammad, S. Shafie, AIP Conf. Proc. 1450, 286 (2012).

[5] A.R.M. Kasim, S. Shafie, Proceeding on Regional Conference on Applied and Engineering Mathematics Vol. 1, No. 20 ( 2010) 124129

[6] P.D. Ariel, International Journal of Engineering Science, 40 (2002) 145-162.

[7] I. Anwar, N. Amin, I. Pop, International Journal of Non-Linear Mechanics, 43 (2008) 814-821.

[8] B. Lehnert, Magnetohydrodynamics: Historical Evolutions and Trends,” Springer, Netherlands, (2007).

[9] J.A. Shercliff, Pergamon Press, Oxford, (1965).

[10] S. Abel, P.H. Veena, K. Rajgopal, V.K. Pravin, International Journal of Non-Linear Mechanics, 39 (2004) 1067-1078.

[11] S. Abel, K.V. Prasad, A. Mahaboob, International Journal of Thermal Sciences, 44 (2005) 465-476.

[12] R. Cortell, Physics Letters A, 357 (2006) 298-305.

[13] V. Aliakbar, A. Alizadeh-Pahlavan, K. Sadeghy, Communications in Nonlinear Science and Numerical Simulation, 14 (2009) 779-794.

[14] A.M. Salem, Physics Letters A, 369 (2007) 315-322.

[15] T. Hayat, M. Mustafa, I. Pop, Communications in Nonlinear Science and Numerical Simulation, 15 (2010) 1183-1196.

[16] M.M. Nandeppanavar, M.S. Abel, K. Vajravelu, International Journal of Heat and Mass Transfer, 53 (2010) 4707-4713.

[17] B. Raftari, K. Vajravelu, Communications in Nonlinear Science and Numerical Simulation, 17 (2012) 4149-4162.

[18] T.R. Mahapatra, A.S. Gupta, International Journal of Non-Linear Mechanics, 39 (2004) 811-820. 